University of Zurich

Department of Economics

Working Paper Series

ISSN 1664-7041 (print)

ISSN 1664-705X (online)

Working Paper No. 159

\title{
Unique Equilibrium in Rent-Seeking Contests with a Continuum of Types
}

Christian Ewerhart

May 2014 


\section{Unique Equilibrium in Rent-Seeking} Contests with a Continuum of Types

5 Abstract It is shown that rent-seeking contests with continuous and inde6 pendent type distributions possess a unique pure-strategy Nash equilibrium.

7 Keywords Rent-seeking · Private information · Pure-strategy Nash equi8 librium $\cdot$ Existence $\cdot$ Uniqueness

9 JEL Classification C7, D7, D8

\footnotetext{
${ }^{\dagger}$ Department of Economics, University of Zurich, Schönberggasse 1, CH-8001 Zurich, Switzerland; phone: +41-44-6343733; e-mail: christian.ewerhart@econ.uzh.ch.
} 
10

11

\section{Introduction}

While rent-seeking contests with continuous and independent type distributions are quite interesting, basic issues such as existence and uniqueness of a pure-strategy Nash equilibrium (PSNE) have been addressed only partially. Indeed, previous work on the issue of existence focused either on symmetric contests (Fey, 2008; Ryvkin, 2010) or on the case of a continuous technology (Wasser, 2013a, 2013b). Moreover, little general was known about the uniqueness of the equilibrium.

Below, it is shown that in any rent-seeking contest with independent and continuous types, there exists a unique PSNE. ${ }^{1}$ The result holds even when the contest is ex-ante asymmetric, ${ }^{2}$ so that the equilibrium may entail inactive types. ${ }^{3}$ Moreover, no restriction is imposed on the shape of the type distributions. Generally, existence ensures consistency of a model, whereas uniqueness strengthens numerical analyses, theoretical results, and experimental findings.

The rest of the paper is structured as follows. Section 2 describes the set-up. Existence is dealt with in Section 3. Section 4 discusses uniqueness. A numerical illustration can be found in Section 5. Section 6 concludes. An Appendix contains technical lemmas.

\footnotetext{
${ }^{1}$ Uniqueness means here that for any given player, any two PSNE strategies differ at most on a null set. This corresponds to the strongest form of uniqueness for PSNE.

${ }^{2}$ Asymmetry may be reflected, e.g., in heterogeneous distributions of marginal costs or in heterogeneous economies of scale.

${ }^{3}$ Wärneryd (2003) explicitly allows for inactive types in a common-value setting.
} 


\section{${ }_{29} 2$ Set-up}

\footnotetext{
${ }^{4}$ As usual, a simple change of variables allows to capture other types of contest success functions and other forms of uncertainty, e.g., about valuations. Cf. Ryvkin (2010).

${ }^{5}$ As shown in the Appendix, this amounts to the standard definition.
} 
49

\section{Existence}

This section builds on prior work by Fey (2008), Ryvkin (2010), and Wasser (2013a). Existence is shown first for the $\varepsilon$-constrained contest, for $\varepsilon>0$, in which each player $i=1, \ldots, N$ may use only strategies with values in $[\varepsilon, \infty)$.

Lemma 3.1 There is a level of activity $E>0$ such that, for any sufficiently small $\varepsilon>0$, there exists a BNE $\sigma^{\varepsilon}$ in the $\varepsilon$-constrained contest such that each player $i$ 's strategy $\sigma_{i}^{\varepsilon}$ is continuous, monotone, and bounded by E.

Proof. Since costs are strictly increasing and convex, there is an $E>$ 0 such that any $y_{i}>E$ is suboptimal. Moreover, $\bar{\Pi}_{i}$ exhibits decreasing differences in $y_{i}$ and $c_{i}$. Hence, existence of a monotone PSNE $\widetilde{\sigma}^{\varepsilon}$ in the $\varepsilon$ constrained contest follows from Athey (2001, Cor. 2.1). Note now that type $c_{i}$ 's $\varepsilon$-constrained problem, $\max _{y_{i} \geq \varepsilon} \bar{\Pi}_{i}\left(y_{i}, \widetilde{\sigma}_{-i}^{\varepsilon}, c_{i}\right)$, has a unique solution $y_{i}=$ $\sigma_{i}^{\varepsilon}\left(c_{i}\right)$. Indeed, if $\widetilde{\sigma}_{-i}^{\varepsilon}\left(c_{-i}\right) \neq 0$ with positive probability, then $\bar{\Pi}_{i}\left(\cdot, \widetilde{\sigma}_{-i}^{\varepsilon}, c_{i}\right)$ is strictly concave on $[\varepsilon, E]$, while otherwise, the unique solution is $y_{i}=\varepsilon$. Hence, $\sigma_{i}^{\varepsilon}\left(c_{i}\right)=\widetilde{\sigma}_{i}^{\varepsilon}\left(c_{i}\right)$ with probability one, for any $i=1, \ldots, N$. This implies that $\sigma_{i}^{\varepsilon}\left(c_{i}\right)$ is also type $c_{i}$ 's best response to $\sigma_{-i}^{\varepsilon}$, for any $i=1, \ldots, N$, and any $c_{i} \in D_{i}$. Thus, $\sigma^{\varepsilon}=\left(\sigma_{1}^{\varepsilon}, \ldots, \sigma_{N}^{\varepsilon}\right)$ is a BNE in the $\varepsilon$-constrained contest. Clearly, each $\sigma_{i}^{\varepsilon}$ is monotone. Finally, continuity of $\sigma_{i}^{\varepsilon}$ follows from Berge's Theorem, as $\bar{\Pi}_{i}\left(\cdot, \sigma_{-i}^{\varepsilon}, \cdot\right)$ is continuous on the compact set $[\varepsilon, E] \times D_{i}$.

Consider now a sequence $\left\{\varepsilon_{m}\right\}_{m=1}^{\infty}$ such that $\varepsilon_{m} \searrow 0$, and select a BNE $\sigma^{m}$ in the $\varepsilon_{m}$-constrained contest for each $m \in \mathbb{N}$, with the properties specified in the previous lemma.

Lemma 3.2 The sequence $\left\{\sigma^{m}\right\}_{m=1}^{\infty}$ has a uniformly converging subse- 
83 is seen to be positive in the range of $c_{i}$ where $y_{i}=\sigma_{i}^{m}\left(c_{i}\right)>0$. Thus, for $\lambda$

84 large, $y_{i}^{\lambda}$ is weakly increasing in $c_{i}$, which proves the claim.

quence.

Proof. In view of Lemma 3.1 and the Theorem of Arzelà-Ascoli, it suffices to find a $\lambda>0$ such that $\sigma_{i}^{m}$ has everywhere a slope exceeding $-\lambda$ for any $m \in \mathbb{N}$ and any $i$. In terms of the transformed choice variable $y_{i}^{\lambda}=y_{i}+\lambda c_{i}$, a type $c_{i}$ 's expected payoff in $\sigma^{m}$ may be written as

$$
\bar{\Pi}_{i}^{\lambda}\left(y_{i}^{\lambda}, \sigma_{-i}^{m}, c_{i}\right)=\int_{D_{-i}} \frac{\left(y_{i}^{\lambda}-\lambda c_{i}\right) d F_{-i}\left(c_{-i}\right)}{y_{i}^{\lambda}-\lambda c_{i}+\sum_{j \neq i} \sigma_{j}^{m}\left(c_{j}\right)}-c_{i} g_{i}\left(y_{i}^{\lambda}-\lambda c_{i}\right),
$$

provided that $y_{i}^{\lambda}-\lambda c_{i}=y_{i}>0$. Hence, for $\lambda$ sufficiently large, the crosspartial

$$
\begin{aligned}
\frac{\partial^{2} \bar{\Pi}_{i}^{\lambda}}{\partial y_{i}^{\lambda} \partial c_{i}} & =\int_{D_{-i}} \frac{2 \lambda \sum_{j \neq i} \sigma_{j}^{m}\left(c_{j}\right) d F_{-i}\left(c_{-i}\right)}{\left(y_{i}+\sum_{j \neq i} \sigma_{j}^{m}\left(c_{j}\right)\right)^{3}}-g_{i}^{\prime}\left(y_{i}\right)+\underbrace{c_{i} \lambda g_{i}^{\prime \prime}\left(y_{i}\right)}_{\geq 0} \\
& \geq \frac{2 \lambda}{N E} \int_{D_{-i}} \frac{\sum_{j \neq i} \sigma_{j}^{m}\left(c_{j}\right) d F_{-i}\left(c_{-i}\right)}{\left(y_{i}+\sum_{j \neq i} \sigma_{j}^{m}\left(c_{j}\right)\right)^{2}}-g_{i}^{\prime}\left(y_{i}\right) \\
& \geq\left(\frac{2 \lambda \underline{c}_{i}}{N E}-1\right) g_{i}^{\prime}\left(y_{i}\right)
\end{aligned}
$$

By Lemma 3.2, one may assume that $\left\{\sigma^{m}\right\}_{m=1}^{\infty}$ converges uniformly to some $\sigma^{*} \in S$. Next, it is shown that in $\sigma^{*}$, at least one player is active with probability one.

Lemma 3.3 There is some player $i$ such that $\sigma_{i}^{*}\left(c_{i}\right)>0$ with probability one. 
(1)

Proof. Suppose that for each $i$, there is a set $\mathcal{D}_{i} \subseteq D_{i}$ of positive measure such that $\sigma_{i}^{*}\left(c_{i}\right)=0$ for all $c_{i} \in \mathcal{D}_{i}$. Then, by uniform convergence, there exists, for any $\varepsilon>0$, an $m_{0}=m_{0}(\varepsilon)$ such that $\sigma_{i}^{m}\left(c_{i}\right)<\varepsilon$ for any $i$, any $c_{i} \in \mathcal{D}_{i}$, and any $m \geq m_{0}$. But, from the Kuhn-Tucker condition for type $c_{i}$ in the $\varepsilon_{m}$-constrained contest,

$$
0 \geq \int_{\mathcal{D}_{-i}} \frac{\sum_{j \neq i} \sigma_{j}^{m}\left(c_{j}\right) d F_{-i}\left(c_{-i}\right)}{\left(\sigma_{i}^{m}\left(c_{i}\right)+\sum_{j \neq i} \sigma_{j}^{m}\left(c_{j}\right)\right)^{2}}-c_{i} g_{i}^{\prime}(E)
$$

where $\mathcal{D}_{-i}=\prod_{j \neq i} \mathcal{D}_{j}$. Integrating over $\mathcal{D}_{i}$, and subsequently summing over $i=1, \ldots, N$, one obtains

$$
0 \geq \int_{\mathcal{D}} \frac{(N-1) d F(c)}{\sum_{i=1}^{N} \sigma_{i}^{m}\left(c_{i}\right)}-\sum_{i=1}^{N} g_{i}^{\prime}(E) \int_{\mathcal{D}_{i}} c_{i} d F_{i}\left(c_{i}\right),
$$

where $\mathcal{D}=\prod_{i=1}^{N} \mathcal{D}_{i}$ and $d F(c)=\prod_{i=1}^{N} d F_{i}\left(c_{i}\right)$. For $\varepsilon$ small, however, this is impossible.

The following is the first main result of this paper.

Theorem 3.4 In the unconstrained contest, $\sigma^{*}$ is a PSNE in continuous and monotone strategies.

Proof. Fix a player $i \in\{1, \ldots, N\}$. For any $m \in \mathbb{N}$, since $\sigma^{m}$ is a $\mathrm{BNE}$ in the $\varepsilon_{m}$-constrained contest, $\bar{\Pi}_{i}\left(\sigma_{i}^{m}\left(c_{i}\right), \sigma_{-i}^{m}, c_{i}\right) \geq \bar{\Pi}_{i}\left(y_{i}, \sigma_{-i}^{m}, c_{i}\right)$ for any $c_{i} \in D_{i}$ and any $y_{i} \geq \varepsilon_{m}$. Therefore, if the event $\sigma_{-i}^{*}\left(c_{-i}\right)=0$ is null, letting $m \rightarrow \infty$ implies $\bar{\Pi}_{i}\left(\sigma_{i}^{*}\left(c_{i}\right), \sigma_{-i}^{*}, c_{i}\right) \geq \bar{\Pi}_{i}\left(y_{i}, \sigma_{-i}^{*}, c_{i}\right)$ for any $c_{i} \in D_{i}$ and any $y_{i}>0$. Suppose next that $\sigma_{-i}^{*}\left(c_{-i}\right)=0$ with positive probability. Then, by Lemma $3.3, \sigma_{i}^{*}\left(c_{i}\right)>0$ with probability one. Let $c_{i} \in D_{i}$ with 
$\sigma_{i}^{*}\left(c_{i}\right)>0$. If $y_{i}>0$, then the argument proceeds as above. To complete the proof, note that $\bar{\Pi}_{i}\left(\cdot, \sigma_{-i}^{*}, c_{i}\right)$ is l.s.c., so that $y_{i}=0$ cannot be the only profitable deviation for $c_{i}$.

\section{Uniqueness}

Consider two PSNE $\sigma^{*}$ and $\sigma^{* *}$ such that, for some player $i$, the event $\sigma_{i}^{*}\left(c_{i}\right) \neq$ $\sigma_{i}^{* *}\left(c_{i}\right)$ has positive probability. Then, as noted below, $\sigma^{*}$ and $\sigma^{* *}$ must differ in an essential way for at least two players.

Lemma 4.1 There are players $i \neq j$ such that each of the independent events $\sigma_{i}^{*}\left(c_{i}\right) \neq \sigma_{i}^{* *}\left(c_{i}\right)$ and $\sigma_{j}^{*}\left(c_{j}\right) \neq \sigma_{j}^{* *}\left(c_{j}\right)$ has positive probability.

Proof. Suppose there is some $i$ such that $\sigma_{-i}^{*}\left(c_{-i}\right)=\sigma_{-i}^{* *}\left(c_{-i}\right)$ with probability one. Then, $\bar{\Pi}_{i}\left(\cdot, \sigma_{-i}^{*}, c_{i}\right)=\bar{\Pi}_{i}\left(\cdot, \sigma_{-i}^{* *}, c_{i}\right)$ for any $c_{i} \in D_{i}$. Thus, $\sigma_{i}^{*}\left(c_{i}\right)=\sigma_{i}^{* *}\left(c_{i}\right)$ with probability one, which is a contradiction.

The following is the second main result of this paper.

Theorem 4.2 The PSNE in the unconstrained contest is unique.

Proof. Following Rosen (1965), write $\sigma^{*, s}=(1-s) \sigma^{*}+s \sigma^{* *}$ for $0 \leq s \leq 1$, and consider

$$
\Phi_{s}=\sum_{i=1}^{N} \int_{D_{i}} \bar{\pi}_{i}\left(\sigma^{*, s}, c_{i}\right)\left(\sigma_{i}^{* *}\left(c_{i}\right)-\sigma_{i}^{*}\left(c_{i}\right)\right) d F_{i}\left(c_{i}\right)
$$

for $s=0,1$, where $\bar{\pi}_{i}\left(\sigma, c_{i}\right)=\partial \bar{\Pi}_{i}\left(\sigma_{i}\left(c_{i}\right), \sigma_{-i}, c_{i}\right) / \partial y_{i}$ denotes type $c_{i}$ 's marginal expected payoff at a profile $\sigma \in S .^{6}$ From the Kuhn-Tucker con-

\footnotetext{
${ }^{6} \mathrm{It}$ is shown in the Appendix that $\Phi_{0}$ and $\Phi_{1}$ are well-defined.
} 
where $z_{i}\left(c_{i}\right)=\sigma_{i}^{* *}\left(c_{i}\right)-\sigma_{i}^{*}\left(c_{i}\right)$. An application of the chain rule delivers

$$
\frac{\partial \pi_{i}\left(\sigma^{*, s}, c_{i}, c_{-i}\right)}{\partial s}=\sum_{j=1}^{N} \frac{\partial^{2} p_{i}\left(\sigma_{i}^{*, s}\left(c_{i}\right), \sigma_{-i}^{*, s}\left(c_{-i}\right)\right)}{\partial y_{i} \partial y_{j}} z_{j}\left(c_{j}\right)-c_{i} \underbrace{g_{i}^{\prime \prime}\left(\sigma_{i}^{*, s}\left(c_{i}\right)\right)}_{\geq 0} z_{i}\left(c_{i}\right),
$$

for any $i$, any $c_{i} \in D_{i}$, and any $c_{-i} \in D_{-i}$. It follows that

$$
\Phi_{1}-\Phi_{0} \leq \int_{D}\left(\int_{0}^{1}\left(\sum_{i=1}^{N} \sum_{j=1}^{N} \frac{\partial^{2} p_{i}\left(\sigma_{i}^{*, s}\left(c_{i}\right), \sigma_{-i}^{*, s}\left(c_{-i}\right)\right)}{\partial y_{i} \partial y_{j}} z_{i}\left(c_{i}\right) z_{j}\left(c_{j}\right)\right) d s\right) d F(c) .
$$


One can verify, however, that

141

$$
\begin{aligned}
& \sum_{i=1}^{N} \sum_{j=1}^{N} \frac{\partial^{2} p_{i}\left(y_{i}, y_{-i}\right)}{\partial y_{i} \partial y_{j}} z_{i} z_{j} \\
= & -\sum_{i=1}^{N} \frac{2 Y_{-i}}{Y^{3}} z_{i}^{2}+\sum_{i=1}^{N} \sum_{j \neq i} \frac{Y-2 Y_{-i}}{Y^{3}} z_{i} z_{j} \\
= & -\frac{2}{Y^{3}} \sum_{i=1}^{N} Y_{-i} z_{i}^{2}-\frac{2}{Y^{3}} \sum_{i=1}^{N} \sum_{j>i} \sum_{k \neq i, j} y_{k} z_{i} z_{j}
\end{aligned}
$$$$
=-\frac{1}{Y^{3}} \sum_{i=1}^{N} Y_{-i} z_{i}^{2}-\frac{1}{Y^{3}} \sum_{i=1}^{N} \sum_{j=1}^{N} \sum_{k \neq i, j} y_{k} z_{i} z_{j}
$$$$
=-\frac{1}{Y^{3}} \sum_{i=1}^{N}\left(z_{i}^{2} Y_{-i}+y_{i} Z_{-i}^{2}\right) \leq 0
$$

146 for any $\left(y_{1}, \ldots, y_{N}\right) \in \mathbb{R}_{+}^{N} \backslash\{0\}$ and any $\left(z_{1}, \ldots, z_{N}\right) \in \mathbb{R}^{N}$, where $Y=\sum_{i=1}^{N} y_{i}$, ${ }_{147} Y_{-i}=\sum_{j \neq i} y_{j}$, and $Z_{-i}=\sum_{j \neq i} z_{j}$. Moreover, $z_{i}^{2} Y_{-i}=z_{i}\left(c_{i}\right)^{2} \sum_{j \neq i} \sigma_{j}^{*, s}\left(c_{j}\right)$ is positive for any $s \in(0,1)$ if $\sigma_{i}^{*}\left(c_{i}\right) \neq \sigma_{i}^{* *}\left(c_{i}\right)$ and $\sigma_{j}^{*}\left(c_{j}\right) \neq \sigma_{j}^{* *}\left(c_{j}\right)$ for some $j \neq i$. Thus, by Lemma $4.1, \Phi_{1}-\Phi_{0}<0$.

\section{Numerical illustration}

Figure 1 shows PSNE strategies in a two-player lottery contest, where types are distributed uniformly on $D_{1}=[0.01,1.01]$ and $D_{2}=[0.51,5.51]$, respectively. Note that player 2 remains inactive for $c_{2}>c_{2}^{*} \approx 4.21$. 

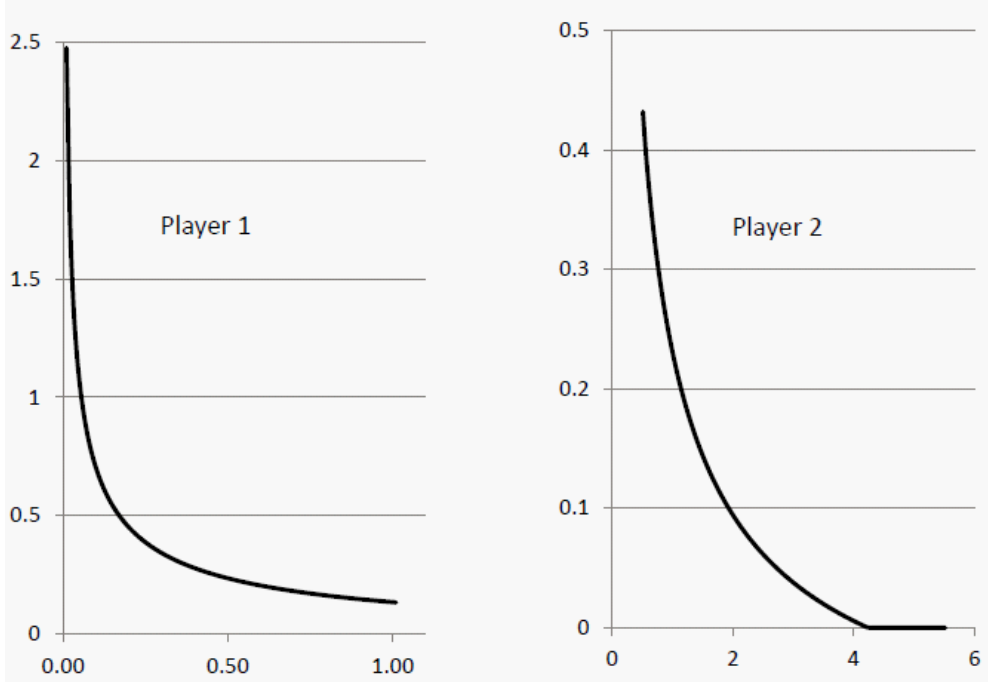

Figure 1: An equilibrium involving inactive types

\section{Concluding remark}

While this paper has focused on the existence and uniqueness of a PSNE in asymmetric rent-seeking contests, it follows from the proofs that also any of the BNE studied by Fey (2008) and Ryvkin (2010) is unique.

\section{Appendix: Technical lemmas}

Lemma A.1 A profile $\sigma^{*} \in S$ is a PSNE in the unconstrained contest if and only if $\int_{D} \Pi_{i}\left(\sigma_{i}^{*}\left(c_{i}\right), \sigma_{-i}^{*}\left(c_{-i}\right), c_{i}\right) d F(c) \geq \int_{D} \Pi_{i}\left(\widehat{\sigma}_{i}\left(c_{i}\right), \sigma_{-i}^{*}\left(c_{-i}\right), c_{i}\right) d F(c)$ for any $i=1, \ldots, N$, and any $\widehat{\sigma}_{i} \in S_{i}$.

Proof. Let $\sigma^{*}$ be a PSNE, and consider a deviation $\widehat{\sigma}_{i} \in S_{i}$ for some player $i$. Then, $\bar{\Pi}_{i}\left(\sigma_{i}^{*}\left(c_{i}\right), \sigma_{-i}^{*}, y_{i}\right) \geq \bar{\Pi}_{i}\left(\widehat{\sigma}_{i}\left(c_{i}\right), \sigma_{-i}^{*}, c_{i}\right)$ for almost any $c_{i} \in D_{i}$. Integrating over $D_{i}$, the assertion follows via Fubini's theorem. Conversely, 
suppose that $\sigma^{*}$ is not a PSNE. Then, there is a player $i$ and a set $\mathcal{D}_{i} \subseteq D_{i}$ of positive measure such that $\sigma_{i}^{*}\left(c_{i}\right)$ is not a best response to $\sigma_{-i}^{*}$ for $c_{i}$, for any $c_{i} \in \mathcal{D}_{i}$. Define $\widehat{\sigma}_{i}\left(c_{i}\right)$ as $c_{i}$ 's best response to $\sigma_{-i}^{*}$ if it exists; otherwise as $\sigma_{i}^{*}\left(c_{i}\right) / 2$ if $\sigma_{i}^{*}\left(c_{i}\right)>0$, and as $\operatorname{pr}\left\{\sigma_{-i}^{*}\left(c_{-i}\right)=0\right\} /\left(2 \bar{c}_{i} g_{i}^{\prime}(E)\right)$ if $\sigma_{i}^{*}\left(c_{i}\right)=0$. Then $\widehat{\sigma}_{i}$ is a profitable deviation.

Lemma A.2 Let $\sigma^{*} \in S$ be a PSNE in the unconstrained contest. Then, for almost any $c_{i} \in D_{i}$, the function $\pi_{i}\left(\sigma^{*}, c_{i}, \cdot\right)$ is integrable, with $\bar{\pi}_{i}\left(\sigma^{*}, c_{i}\right)=$ $\int_{D_{-i}} \pi_{i}\left(\sigma^{*}, c_{i}, c_{-i}\right) d F_{-i}\left(c_{-i}\right)$. Moreover, $\bar{\pi}_{i}\left(\sigma^{*}, \cdot\right)$ is integrable.

Proof. The first claim is obvious if $\sigma_{i}^{*}\left(c_{i}\right)>0$ for almost any $c_{i} \in D_{i}$. Suppose that $\sigma_{i}^{*}\left(c_{i}\right)=0$ with positive probability. Then, by Lemma 3.3, the event $\sigma_{-i}^{*}\left(c_{-i}\right)=0$ is null. Take some $c_{-i} \in D_{-i}$ with $\sigma_{-i}^{*}\left(c_{-i}\right) \neq 0$. Then, for any $c_{i} \in D_{i}$, by concavity, the difference quotient $\Pi_{i}\left(y_{i}, \sigma_{-i}^{*}\left(c_{-i}\right), c_{i}\right) / y_{i}$ is monotone increasing as $y_{i} \searrow 0$, with limit $\pi_{i}\left(\sigma^{*}, c_{i}, c_{-i}\right)$. Since also $\Pi_{i}\left(y_{i}, \sigma_{-i}^{*}\left(c_{-i}\right), c_{i}\right) / y_{i} \geq-\bar{c}_{i} g_{i}^{\prime}(E)$, the first claim follows from Levi's theorem. The second claim follows from Lebesgue's theorem, because $\bar{\pi}_{i}\left(\sigma^{*}, \cdot\right) \leq 0$ from the Kuhn-Tucker conditions, and because $\bar{\pi}_{i}\left(\sigma^{*}, \cdot\right) \geq-\bar{c}_{i} g_{i}^{\prime}(E)$, as above.

\section{References}

[1] Athey, S., 2001. Single crossing properties and the existence of pure strategy equilibria in games of incomplete information, Econometrica 69(4), $861-889$.

[2] Fey, M., 2008. Rent-seeking contests with incomplete information. Public Choice 135(3), 225-236. 
[3] Rosen, J.B., 1965. Existence and uniqueness of equilibrium points for concave $N$-person games. Econometrica 33(3), 520-534.

[4] Ryvkin, D., 2010. Contests with private costs: Beyond two players. European Journal of Political Economy 26(4), 558-567.

[5] Wärneryd, K., 2003. Information in conflicts. Journal of Economic Theory 110(1), 121-136.

[6] Wasser, C., 2013a. A note on Bayesian Nash equilibria in imperfectly discriminating contests. Mathematical Social Sciences 66(2), 180-182.

[7] Wasser, C., 2013b. Incomplete information in rent-seeking contests. Economic Theory 53(1), 239-268. 